\title{
Study of twist-2 distribution amplitudes and the decay constants of pseudoscalar and vector heavy mesons in light-front quark model
}

\author{
Nisha Dhiman* ${ }^{*}$ \\ Department of Physics, Dr. B. R. Ambedkar National Institute of Technology, Jalandhar-144011, \\ India \\ E-mail: nishdhiman1292@gmail.com
}

\section{Harleen Dahiya}

Department of Physics, Dr. B. R. Ambedkar National Institute of Technology, Jalandhar-144011, India

\section{Chueng-Ryong Ji}

Department of Physics, North Carolina State University, Raleigh, North Carolina 27695-8202, USA

\section{Ho-Meoyng Choi}

Department of Physics, Teachers College, Kyungpook National University, Daegu, Korea 41566

\begin{abstract}
We study the twist-2 distribution amplitudes (DAs) and the decay constants of pseudoscalar as well as the longitudinally and transversely polarized vector heavy $\left(D, D_{s}, B, B_{s}\right)$ mesons in the light-front quark model with the Coulomb plus exponential-type confining potential $V_{\exp }=a+$ $b e^{\alpha r}$ in addition to the hyperfine interaction. We first compute the mass spectra of ground state pseudoscalar and vector heavy mesons and fix the model parameters necessary for the analysis, applying the variational principle with the trial wave function up to the first three lowest order harmonic oscillator (HO) wave functions $\Phi\left(x, \mathbf{k}_{\perp}\right)=\sum_{n=1}^{3} c_{n} \phi_{n}$. We then obtain the numerical results for the corresponding decay constants. We analyze the variation of DAs as a function of momentum fraction. We also compare our results with the available experimental data as well as with the other theoretical model predictions.
\end{abstract}

Light Cone 2019 - QCD on the light cone: from hadrons to heavy ions - LC2019

16-20 September 2019

Ecole Polytechnique, Palaiseau, France

\footnotetext{
*Speaker.

${ }^{\dagger}$ N. D. would like to thank Monika Randhawa (University Institute of Engineering and Technology, PU, Chandigarh, India) for helpful discussions.
} 


\section{Introduction}

The nonperturbative structure of the hadron is well described by the hadronic or quark distribution amplitudes (DAs) which not only encode important information on bound states in QCD but also play an essential role in describing the various hard exclusive processes $[1,2]$ of QCD via the factorization theorem in analogous to parton distributions in inclusive processes. They also help in understanding the distribution of partons in terms of the longitudinal momentum fraction as they are the longitudinal projection of the hadronic wave functions obtained by integrating out the transverse momenta of the fundamental constituents of the hadron. Hadronic DAs are defined in terms of vacuum-to-hadron matrix elements of particular non-local quark or quark-gluon operators. The lowest moments of the hadronic DAs for a quark and an antiquark inside a meson provide us the knowledge of decay constants that are considered as direct source of information on the Cabibbo-Kobayashi-Maskawa (CKM) matrix elements, i.e. the fundamental parameters of the $\mathrm{SM}$. The precise determination of decay constants will further allow us to test the unitarity of the quark mixing matrix and CP violation in the SM [3].

The present work is focused on the study of the ground state pseudoscalar and vector heavy $(D$, $\left.D^{*}, D_{s}, D_{s}^{*}, B, B^{*}, B_{s}, B_{s}^{*}\right)$ mesons mass spectra. The decay constants and the twist-2 DAs of pseudoscalar, longitudinally and transversely polarized vector heavy mesons have been studied in detail using the light-front quark model (LFQM) based on the idea of modelling the potential. This study will not only provide essential informations on the understanding of the universal nonperturbative quantities but also help further analyses of the hard exclusive processes.

\section{Light-front quark model}

Our LFQM is based on the idea that we consider the radial wave function $\Phi\left(x, \mathbf{k}_{\perp}\right)$ as a trial wave function for the variational principle to the QCD-motivated Hamiltonian $[4,5,6]$

$$
H_{\mathrm{c} . \mathrm{m} .}=\sqrt{\mathbf{k}^{2}+m_{q}^{2}}+\sqrt{\mathbf{k}^{2}+m_{\bar{q}}^{2}}+V_{q \bar{q}}
$$

where $V_{q \bar{q}}$ is the effective interaction potential between quark and antiquark in the rest frame of the meson which is given by Coulomb $\left(V_{\text {Coul }}\right)$ plus exponential-type potential $\left(V_{\exp }\right)$ in addition to the hyperfine interaction $\left(V_{\text {hyp }}\right)$. That is,

$$
\begin{aligned}
V_{q \bar{q}} & =V_{\exp }+V_{\text {Coul }}+V_{\text {hyp }} \\
& =a+b e^{\alpha r}-\frac{4 \kappa}{3 r}+\frac{2}{3} \frac{\mathbf{S}_{\mathbf{q}} \cdot \mathbf{S}_{\overline{\mathbf{q}}}}{m_{q} m_{\bar{q}}} \nabla^{2} V_{\text {Coul }},
\end{aligned}
$$

where $a, b$ and $\alpha$ are the parameters of the potential, $\kappa$ is the strong coupling constant which has been taken as one of the variation parameter in this work, $\left\langle\mathbf{S}_{\mathbf{q}} \cdot \mathbf{S}_{\overline{\mathbf{q}}}\right\rangle=-3 / 4(1 / 4)$ for the pseudoscalar (vector) meson, respectively. We use the radial wave function $\Phi\left(x, \mathbf{k}_{\perp}\right)$ as an expansion of the true wave function in the three lowest order HO wave functions $\Phi\left(x, \mathbf{k}_{\perp}\right)=\sum_{n=1}^{3} c_{n} \phi_{n S}$ for both pseudoscalar and vector mesons [7]. The optimized values of the constituent quark masses and other potential parameters that give the best fit of the ground state mass spectra of mesons are summarized in Table 1. 
Table 1: Constituent quark masses and the potential parameters $\sigma, \alpha$, and $a$ (in units of $\mathrm{GeV}$ ) obtained by the variational principle for the Hamiltonian with a smeared-out hyperfine interaction. Here $q=u$ and $d$.

\begin{tabular}{lllllll}
\hline \hline$m_{q}$ & $m_{s}$ & $m_{c}$ & $m_{b}$ & $\sigma$ & $\alpha$ & $a$ \\
\hline 0.202 & 0.405 & 1.725 & 5.182 & 0.451 & 0.15 & -1.075 \\
\hline \hline
\end{tabular}

Due to the presence of hyperfine interaction in our variational process, we have different sets of $\beta$ values for pseudoscalar and vector mesons, respectively. The optimal Gaussian parameters $\beta_{q \bar{q}}^{P}$ and $\beta_{q \bar{q}}^{V}$ for pseudoscalar and vector mesons obtained by the variational principle are listed in Table 2. Using these fixed model parameters, we obtained the ground state pseudoscalar $\left(D_{(s)}, B_{(s)}\right)$ and

Table 2: The Gaussian parameter $\beta(\mathrm{GeV})$ for ground state pseudoscalar $\left(D, D_{s}, B, B_{s}\right)$ and vector $\left(D^{*}, D_{s}^{*}\right.$, $B^{*}, B_{s}^{*}$ ) mesons obtained by the variational principle. Here $q=u$ and $d$.

\begin{tabular}{lllll}
\hline \hline$J^{P C}$ & $\beta_{q c}$ & $\beta_{c s}$ & $\beta_{q b}$ & $\beta_{b s}$ \\
\hline $0^{-+}$ & 0.2980 & 0.3010 & 0.3191 & 0.3290 \\
$1^{--}$ & 0.2818 & 0.2926 & 0.3115 & 0.3250 \\
\hline \hline
\end{tabular}

vector $\left(D_{(s)}^{*}, B_{(s)}^{*}\right)$ meson mass spectra. Our results are summarized in Table 3, comparing with the experimental data [8] and the previous results obtained from the linear and HO potentials $[9,10]$.

Table 3: Ground state mass spectra (in units of $\mathrm{GeV}$ ) of pseudoscalar $\left(D_{(s)}, B_{(s)}\right)$ and vector $\left(D_{(s)}^{*}, B_{(s)}^{*}\right)$ mesons obtained from the exponential type potential and their comparison with the experimental data [8] and the LFQM results obtained from the linear and HO potentials $[9,10]$.

\begin{tabular}{lcccccccc}
\hline \hline & $M_{D}$ & $M_{D^{*}}$ & $M_{D_{s}}$ & $M_{D_{s}^{*}}$ & $M_{B}$ & $M_{B^{*}}$ & $M_{B_{s}}$ & $M_{B_{s}^{*}}$ \\
\hline Present work & 1.803 & 1.884 & 1.929 & 1.971 & 5.212 & 5.242 & 5.313 & 5.329 \\
Exp. [8] & 1.869 & 2.010 & 1.968 & 2.112 & 5.279 & 5.325 & 5.367 & 5.415 \\
LFQM, Lin [9] & 1.836 & 1.998 & 2.011 & 2.109 & 5.235 & 5.315 & 5.375 & 5.424 \\
LFQM, HO [9] & 1.821 & 2.024 & 2.005 & 2.150 & 5.235 & 5.349 & 5.378 & 5.471 \\
LFQM [10] & 1.875 & 1.962 & 1.981 & 2.031 & 5.233 & 5.268 & 5.314 & 5.333 \\
\hline \hline
\end{tabular}

\section{Numerical Results}

\subsection{Decay Constants}

For the present calculations of the decay constants, we include the systematic errors in our analysis obtained both from the $\pm 10 \%$ variation of $\beta$ values for the fixed quark masses and the $\pm 10 \%$ variation of quark masses for the fixed $\beta$ values. In Tables 4 and 5, we present our predictions for the decay constants of pseudoscalar, longitudinally, and transversely polarized vector $D$ and $B$ mesons, respectively. 
Table 4: Pseudoscalar, longitudinally and transversely polarized vector $D$ meson decay constants (in units of $\mathrm{MeV}$ ) in the present work and their comparison with the available experimental data and other theoretical model predictions.

\begin{tabular}{lcccccc}
\hline \hline & $f_{D}$ & $f_{D^{*}}$ & $f_{D^{*}}^{\perp}$ & $f_{D_{s}}$ & $f_{D_{s}^{*}}$ & $f_{D_{s}^{*}}^{\perp}$ \\
\hline Present work & $197_{-20-1.0}^{+19+0.2}$ & $230_{-28+6}^{+29-5}$ & $208_{-24+3}^{+24-3}$ & $219_{-22-0.8}^{+21-0.2}$ & $253_{-31+6}^{+31-6}$ & $233_{-26+3}^{+26-3}$ \\
Exp. [8] & $203.7 \pm 4.7$ & - & - & $257.8 \pm 4.1$ & - & - \\
LFQM, Lin [9] & 197 & 239 & - & 233 & 274 & - \\
LFQM, HO [9] & 180 & 212 & - & 218 & 252 & - \\
LFQM, Lin [10] & 208 & 230 & - & 231 & 260 & - \\
\hline \hline
\end{tabular}

Table 5: Pseudoscalar, longitudinally and transversely polarized vector $B$ meson decay constants (in units of $\mathrm{MeV}$ ) in the present work and their comparison with the available experimental data and other theoretical model predictions.

\begin{tabular}{lcccccc}
\hline \hline & $f_{B}$ & $f_{B^{*}}$ & $f_{B^{*}}^{\perp}$ & $f_{B_{s}}$ & $f_{B_{s}^{*}}$ & $f_{B_{s}^{*}}^{+}$ \\
\hline Present work & $163_{-20+4}^{+21-4}$ & $172_{-23+6}^{+24-6}$ & $165_{-21+5}^{+22-5}$ & $184_{-23+4}^{+23-4}$ & $194_{-25+7}^{+26-6}$ & $187_{-24+6}^{+24-5}$ \\
Exp. [8] & $188 \pm 25$ & - & - & - & - & - \\
LFQM, Lin [9] & 171 & 186 & - & 205 & 220 & - \\
LFQM, HO [9] & 161 & 173 & - & 208 & 223 & - \\
LFQM, Lin [10] & 181 & 188 & - & 205 & 216 & - \\
\hline \hline
\end{tabular}

\subsection{Quark Distribution Amplitudes}

The quark DAs are defined in terms of the matrix elements of non-local operators that are sandwitched between the vacuum and the meson states [11]. The explicit forms of quark DAs in our LFQM are given by [12]

$$
\begin{aligned}
& \phi_{P}(x)=\frac{2 \sqrt{6}}{f_{P}} \int \frac{d^{2} \mathbf{k}_{\perp}}{\sqrt{16 \pi^{3}}} \sqrt{\frac{\partial k_{z}}{\partial x}} \Phi\left(x, \mathbf{k}_{\perp}\right) \frac{\mathscr{A}}{\sqrt{\mathscr{A}^{2}+\mathbf{k}_{\perp}^{2}}}, \\
& \phi_{V \|}(x)=\frac{2 \sqrt{6}}{f_{V}} \int \frac{d^{2} \mathbf{k}_{\perp}}{\sqrt{16 \pi^{3}}} \sqrt{\frac{\partial k_{z}}{\partial x}} \frac{\Phi\left(x, \mathbf{k}_{\perp}\right)}{\sqrt{\mathscr{A}^{2}+\mathbf{k}_{\perp}^{2}}}\left\{\mathscr{A}+\frac{2 \mathbf{k}_{\perp}^{2}}{M_{0}+m_{q}+m_{\bar{q}}}\right\}, \\
& \phi_{V \perp}(x)=\frac{2 \sqrt{6}}{f_{V}^{\perp}} \int \frac{d^{2} \mathbf{k}_{\perp}}{\sqrt{16 \pi^{3}}} \sqrt{\frac{\partial k_{z}}{\partial x}} \frac{\Phi\left(x, \mathbf{k}_{\perp}\right)}{\sqrt{\mathscr{A}^{2}+\mathbf{k}_{\perp}^{2}}}\left\{\mathscr{A}+\frac{\mathbf{k}_{\perp}^{2}}{M_{0}+m_{q}+m_{\bar{q}}}\right\},
\end{aligned}
$$

Our results for the quark DAs of $D, D^{*}, D_{s}$ and $D_{s}^{*}$ mesons show much broader shapes than those of $B, B^{*}, B_{s}$ and $B_{s}^{*}$ mesons due to the large mass difference between $b$ and $c$ quarks. 

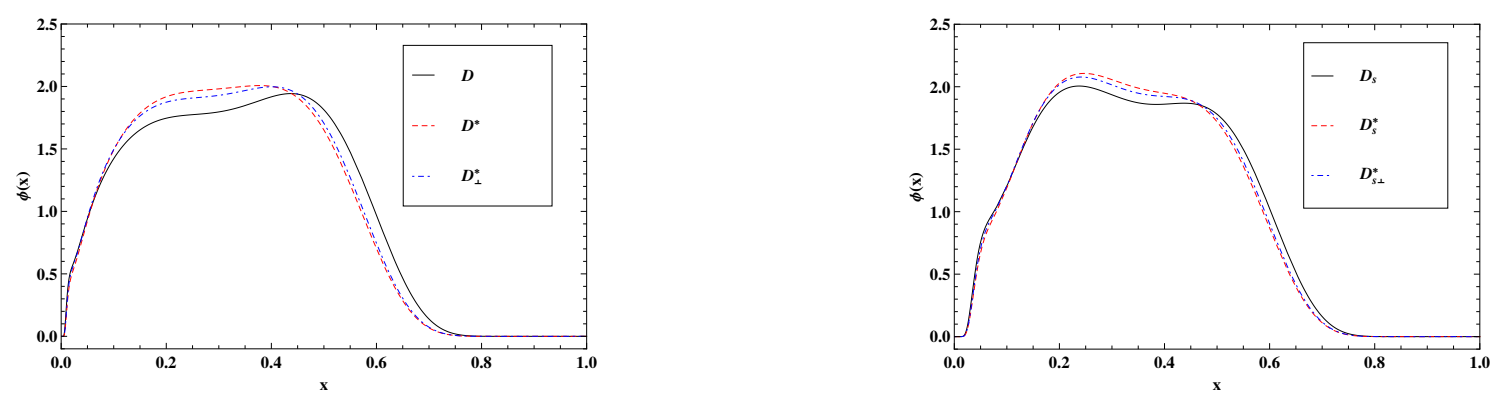

Figure 1: Normalized DAs for the heavy pseudoscalar (solid line), longitudinally (dashed line) and transversely (dotted dashed line) polarized vector $D$ (left panel) and $D_{s}$ (right panel) mesons.
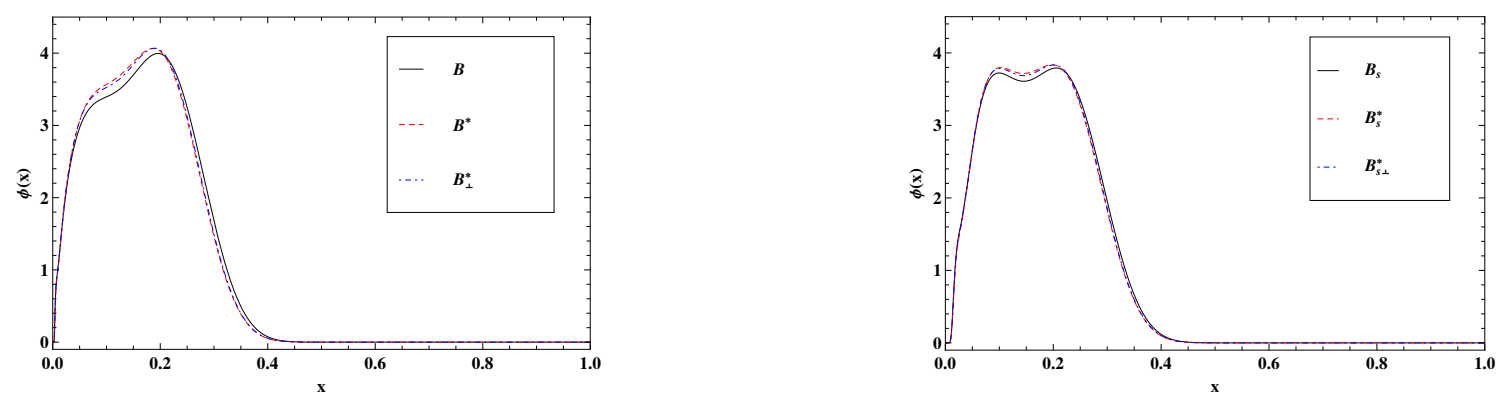

Figure 2: Normalized DAs for the heavy pseudoscalar (solid line), longitudinally (dashed line) and transversely (dotted dashed line) polarized vector $B$ (left panel) and $B_{s}$ (right panel) mesons.

\section{References}

[1] S. J. Brodsky and G. P. Lepage, Contribution to 'Perturbative Quantum Chromodynamics', A. H. Mueller (Ed.). World Scientific Publ. Co. Adv. Ser. Direct. High Energy Phys. 5, 93 (1989).

[2] V. L. Chernyak and A. R. Zhitnitsky, Nucl. Phys. B 201, 492 (1982); 214, 547 (1983).

[3] M. Blanke, TTP17-017 Lectures given at the ESHEP 2016 Summer School in Skeikampen, Norway, June 15-28, 2016.

[4] H.-M. Choi and C.-R. Ji, Phys. Rev. D 80, 054016 (2009).

[5] H.-M. Choi and C.-R. Ji, Phys. Rev. D 59, 074015 (1999).

[6] H.-M. Choi and C.-R. Ji, Phys. Lett. B 460, 461 (1999).

[7] N. Dhiman, H. Dahiya, C.-R. Ji, and H.-M. Choi, Phys. Rev. D 100, 014026 (2019).

[8] M. Tanabashi et al., (Particle Data Group), Phys. Rev. D 98, 030001 (2018).

[9] H.-M. Choi, Phys. Rev. D 75, 073016 (2007).

[10] H.-M. Choi, C.-R. Ji, Z. Li, and H.-Y. Ryu, Phys. Rev. C 92, 055203 (2015).

[11] C.-W. Hwang, Phys. Rev. D 81, 114024 (2010).

[12] H.-M. Choi and C.-R. Ji, Phys. Rev. D 75, 034019 (2007). 